\title{
Combined biomarker for prediction of response to an immune checkpoint inhibitor in metastatic gastric cancer
}

\section{You Jeong Heo ${ }^{1}$, So Young Kang ${ }^{2}$, Seung Tae Kim³, Won Ki Kang ${ }^{3}$, Jeeyun Lee ${ }^{3}$, Kyoung-Mee Kim²}

\author{
${ }^{1}$ The Samsung Advanced Institute for Health Sciences \& Technology (SAIHST), Samsung Medical Center, Sungkyunkwan \\ University School of Medicine, Seoul, Korea \\ ${ }^{2}$ Department of Pathology \& Translational Genomics, Samsung Medical Center, Sungkyunkwan University School of Medicine, \\ Seoul, Korea \\ ${ }^{3}$ Division of Hematology-Oncology, Department of Internal Medicine, Samsung Medical Center, Sungkyunkwan University School \\ of Medicine, Seoul, Korea
}

Received: August 2, 2019

Revised: October 15, 2019

Accepted: October 16, 2019

Corresponding authors:

Jeeyun Lee

Division of Hematology-

Oncology, Department of Internal

Medicine, Samsung Medical

Center, Sungkyunkwan University

School of Medicine, 81 Irwon-ro,

Gangnam-gu, Seoul 06351, Korea

Tel: +82-2-3410-3459

E-mail: jyunlee@skku.edu

Kyoung-Mee Kim

Department of Pathology \&

Translational Genomics, Samsung

Medical Center, Sungkyunkwan

University School of Medicine,

81 Irwon-ro, Gangnam-gu, Seoul

06351, Korea

Tel: +82-2-3410-2768

E-mail:kkmkys@skku.edu

This is an Open Access article distributed under the terms of the Creative Commons Attribution Non-Commercial License (http:// creativecommons.org/licenses/ by-nc/4.0/).

\section{ABSTRACT}

Purpose: Immune checkpoint blockades (ICB) have been successful in gastric cancer (GC). However, the majority of unselected patients with GC fail to respond to ICB. It is crucial to identify precise biomarkers to predict response to ICB.

Methods: Gene expression profiling of formalin-fixed and paraffin-embedded GC tissues from 25 patients treated with ICB (pembrolizumab) targeting programmed cell death protein 1 (PD-1) was performed using NanoString (NanoString Technologies). For development of a gene signature to predict response to ICB, differential gene expression analysis with linear regression modeling was performed with area under the curve packages in $R$.

Results: From the analysis, 10 genes were differentially expressed between patients with response and no response to ICB $(P<0.01)$. To identify a biomarker predicting response to $I C B$, four genes were selected based on $\mid \log _{2}$ (foldchange) $\mid \geq 1$. After calculating the IMmunotherapy Against Gastrlc Cancer (IMAGiC) score, patients were divided into two groups: to be responder and to be non-responder, according to Response Evaluation Criteria in Solid Tumors (RECIST) guidelines. The IMAGiC score was significantly associated with RECIST groups $(P=0.0057)$, Epstein-Barr virus $(P=0.048)$, and tumor mutational load $(P=0.023)$; however, was not significantly correlated with microsatellite instability status $(P=0.14)$ and programmed death ligand 1 (PD-L1) expression $(P=0.095)$. To reproduce IMAGiC with different technology, we retested the results with a quantitative real-time polymerase chain reaction (qRT-PCR) method, and the precision of reproduction of $87.5 \%$. In validation cohort with 17 samples from the ongoing trial with nivolumab, the precision of IMAGiC qRT-PCR was $100 \%$.

Conclusion: Our identified gene signatures and proposed IMAGiC model for predicting response to pembrolizumab in patients with GC showed validity.

Keywords: Biomarkers; Pembrolizumab; Prediction; Response; Stomach neoplasms 


\section{INTRODUCTION}

Gastric cancer (GC) is the most common cancer and the third leading cause of cancer death worldwide [1]. The U.S. Food and Drug Administration (FDA) approved pembrolizumab, an antibody against programmed cell death protein 1 (PD-1), to treat patients with advanced solid tumors bearing the microsatellite unstable (MSI)/DNA mismatch repair (dMMR)-deficient biomarker [2]. A recent nonrandomized, multicenter, multicohort basket trial of pembrolizumab in 475 patients with one of 20 different programmed death ligand 1 (PD-L1) -positive advanced solid tumors showed diverse objective response rates (ORRs) across different cancer types. Biomarkers that can predict response to anti-PD-1 therapy across multiple tumor types include T-cell inflamed gene expression profile (GEP), PD-L1 expression, and/or tumor mutational burden (TMB) [3]. However, larger studies are needed to obtain more precision within individual cancer types and to assess the potential clinical utility of developing cutoffs for such biomarkers in patient selection for anti-PD-1 therapy [3].

In GC, a single-arm, multicohort, pembrolizumab phase 2 trial (KEYNOTE-059) showed an ORR of $11.6 \%$, and $2.3 \%$ of the patients showed complete responses (CRs), with a lower proportion of responders among patients with GC compared to patients with non-small cell lung cancer [4]. The high proportion of non-responders and the emergence of resistance in patients who do initially respond pose significant challenges in the field of cancer immunotherapy [5]. A recent molecular characterization study of metastatic GC patients treated with pembrolizumab showed that MSI, Epstein-Barr virus (EBV)-positivity, PD-L1 combined positive score (CPS) positivity, TMB, and decrease in circulating tumor DNA levels at 6 weeks post-treatment were associated with response to treatment [6]. Although T-cell inflamed GEP has been shown to predict the clinical efficacy of pembrolizumab therapy across a set of 20 diverse solid tumors and has provided biomarkers that are potentially relevant for selection of patients who may benefit from PD-1 inhibition, it did not predict responses in patients with metastatic GC. Chronically inflamed mucosa in ulcerated cancer tissue or mesenchymal phenotype linked to decreased tumor susceptibility to immune effector cells [5] would contribute to the underlying mechanism for the lack of response to immune checkpoint blockade (ICB).

Predictive biomarkers for immunotherapy differ from the traditional biomarkers used for targeted therapies because of the complexity of the immune response and tumor biology. Recently, immuno-predictive score, IMPRES, based on 45 immune checkpoint genes was developed to predict responses to ICB in patients with melanoma [7]. Given the differences in tumor biology and need for clinical-grade biomarkers to guide the choice of agent to maximize the likelihood of patient benefit, we developed a GC-specific gene expression set to predict response to pembrolizumab.

\section{METHODS}

\section{Patient selection}

From 2013 to 2017, 61 patients with mGC were enrolled for prospective single-site, phase 2 clinical trial to assess clinical responses to pembrolizumab [6]. The trial protocol was approved by the Institutional Review Board of Samsung Medical Center (Seoul, Korea) (2015-09-053) of Samsung Medical Center (Seoul, Korea) and all patients provided written informed consent. Among them, 21 cases were selected for the present study based on availability of primary tumor tissue and clinical responses to pembrolizumab. Their median age was 57 years (range, 26 to 78 years), and they included four patients (19.1\%) with MSI GC and two patients (9.5\%) with EBV-positive GC. Clinical data, including demographic features and treatment outcomes, were obtained by reviewing

Table 1. Characteristics of patients $(n=21)$

\begin{tabular}{lc}
\hline Characteristic & Value \\
\hline Age $(y r)$ & $57(26-78)$ \\
Sex & \\
Male & $16(76.2)$ \\
Female & $5(23.8)$ \\
MSI & \\
MSI & $4(19.1)$ \\
MSS & $17(81.0)$ \\
EBV & \\
Positive & $2(9.5)$ \\
Negative & $19(90.5)$ \\
Response & \\
CR & $2(9.5)$ \\
PR & $4(19.1)$ \\
SD & $5(23.8)$ \\
PD & $10(47.6)$ \\
\hline
\end{tabular}

Values are presented as median (range) or number (\%). MSI, microsatellite unstable; MSS, microsatellite stable; EBV, Epstein-Barr virus; $C R$, complete response; $P R$, partial response; $S D$, stable disease; PD, progressive disease. 
medical records. The precise characteristics of the patients with responses to pembrolizumab are described in Table 1.

\section{RNA extraction}

For isolation of total RNA from formalin-fixed paraffin-embedded tissue, 10 (4- $\mu$ m-thick) sections were cut from each tissue block. RNA was isolated using the RNeasy FFPE kit (Qiagen, Hilden, Germany) according to the manufacturer's instructions. The preparations were deparaffinized and subjected to proteinase $\mathrm{K}$ treatment, on-column DNase digestion, and elution using RNase-free water as described in the manufacturer's protocol. Total RNA samples were stored at $-80^{\circ} \mathrm{C}$ until use. RNA concentrations were measured using NanoDrop (Thermo Fisher Scientific, Waltham, MA, USA).

\section{Gene expression profiling using a NanoString assay}

For gene expression profiling, a NanoString assay (NanoString Technologies Inc., Seattle, WA, USA) was performed with GC tissue sample RNA. For this experiment, we made probes containing 168 genes related to mesenchymal signature [8] and host immune response genes identified by comprehensive analyses of tumor immunity [9]. For control, 11 housekeeping genes and 14 technical internal control genes were also added. We performed the NanoString assays following the standard protocol 'Setting up $12 \mathrm{nCounter}$ Assays (MAN-C0003-03, 2008-2013).' Hybridization reactions were incubated for 18 hours. Data analysis was performed using the nSolver software available from NanoString Technologies. Data were normalized using housekeeping genes and internal controls and were transformed into a $\log _{10}$ scale in nSolver software (version 4.0).

\section{Differentially expressed gene analysis for response to pembrolizumab}

For development of a gene signature that predicts response to pembrolizumab, differentially expressed gene (DEG) analysis was performed using nSolver software. To identify DEG by comparing the pembrolizumab response group and no response group, a $\mathrm{P}<0.01$ was chosen as a cutoff value.

\section{Development of a prediction model for response to pembrolizumab and validation in The Cancer Ge- nome Atlas and Asian Cancer Research Group cohorts To construct a prediction model, the mRNA expression levels of genes with significantly different expression patterns and the PD-L1 CPS of the GC tissues were analyzed using a linear regression model. The prediction model was evaluated by cal-}

culating sensitivity and specificity with the area under the curve (AUC). The cutoff values for dividing patients with mGC into the response and no response groups were also defined by the accuracy function in the AUC package. To evaluate our prediction model, cross validation was performed 10 times, and the root mean squared error (RMSE) for each validation was calculated. As MSI and EBV-positivity and TMB are closely related to responses to immunotherapy, we validated our results from RNA sequencing data of The Cancer Genome Atlas (TCGA) and microarray data of Asian Cancer Research Group (ACRG) (Supplementary Fig. 1). The ComBat function was used for adjusting gene expression data using the sva package, because the validation data and test data had different platforms. All statistical analyses and all visualized plots were performed in $\mathrm{R}$ program version 3.4.4 (R Foundation for Statistical Computing, Vienna, Austria). The total mutation rate was calculated with the number of somatic nonsynonymous single nucleotide variant (SNV) mutations per megabase [10], and the threshold for high TMB was set as the upper tertile [6].

\section{Reproduction of test with quantitative real-time poly- merase chain reaction}

To reproduce our test with different platforms of technology, we set up quantitative real-time polymerase chain reaction (qRT-PCR) on a $7900 \mathrm{HT}$ Sequence Detection System (Applied Biosystems, Foster City, CA, USA) in 384-well plates using a final volume of $10 \mu \mathrm{L}$ per reaction consisting of $5 \mu \mathrm{L} 2 \mathrm{X}$ Taqman PreAmp Master Mix, $4 \mu \mathrm{L}$ cDNA sample, and $1 \mu \mathrm{L}$ primer/ probe. PCR amplifications were performed in triplicate wells using the following conditions: 2 minutes at $50^{\circ} \mathrm{C}$ and $10 \mathrm{~min}$ utes at $94^{\circ} \mathrm{C}$, followed by 40 two-temperature cycles of $95^{\circ} \mathrm{C}$ for 15 seconds and $60^{\circ} \mathrm{C}$ for 60 seconds.

\section{Immunohistochemistry for PD-L1}

Immunohistochemistry (IHC) was performed on each representative section of the FFPE samples. Staining for PD-L1 was conducted using an FDA-approved monoclonal mouse antibody, PD-L1 22C3 pharmDx (Dako, Carpinteria, CA, USA). PDL1-stained IHC slides were interpreted by an experienced pathologist (K.M.K.): CPS was calculated by summing the number of PD-L1-stained cells (tumor cells, lymphocytes, macrophages) and dividing the result by the total number of viable tumor cells, multiplying by 100 [11], and assessing according to the PD-L1 IHC 22C3 pharmDx instructions for use (https:// www.agilent.com/cs/library/ usermanuals/public/29219_ pd-l1-ihc-22C3-pharmdx-gastric-interpretation-manual_ us.pdf). PD-L1 IHC was interpreted as positive if the score 
was 1 or more and negative if the score was less than 1.

\section{Statistical analyses}

Statistical analyses were conducted using R software (https:// www.r-project.org/foundation/) version 3.4.4. Pearson correlation coefficient was used to find correlations between IMmunotherapy Against Gastrlc Cancer (IMAGiC) groups with Response Evaluation Criteria in Solid Tumors (RECIST) group, PD-L1 CPS, and EBV infection status. To compare the IMAGiC scores with RECIST groups, EBV status, and tumor mutation lead (TML), Wilcoxon test and Kruskal-Wallis test were used. All P-values were two-sided, and $P<0.05$ was considered significant.

\section{RESULTS}

\section{Differentially expressed genes in responses to pem- brolizumab}

Gene expression profiling from $21 \mathrm{GC}$ tissues were analyzed in relation to responses to pembrolizumab. CR was found in two cases, partial response (PR) in four cases, stable disease (SD) in five cases, and progressive disease (PD) in 10 cases based on the RECIST. These cases were reallocated into two groups based on RECIST: a response group that included the patients with $\mathrm{CR}$ and $\mathrm{PR}$ and a no-response group that included those with SD and PD.

To identify prediction signature genes, DEG analyses were performed using nSolver software (Fig. 1A). Enrichment analysis was performed using all DEGs $(P<0.05)$. All DEGs were most significantly associated with EBV infection and immune responses, including antigen processing and presentation and the innate immune response (Fig. 1B, Supplementary Fig. 2), suggesting that the most important factors in predicting response to pembrolizumab are related to immune reactions. Finally, four genes that were significantly differentially expressed between the response group and the no response group for pembrolizumab therapy were selected in the DEG analysis $(P<0.01)$.
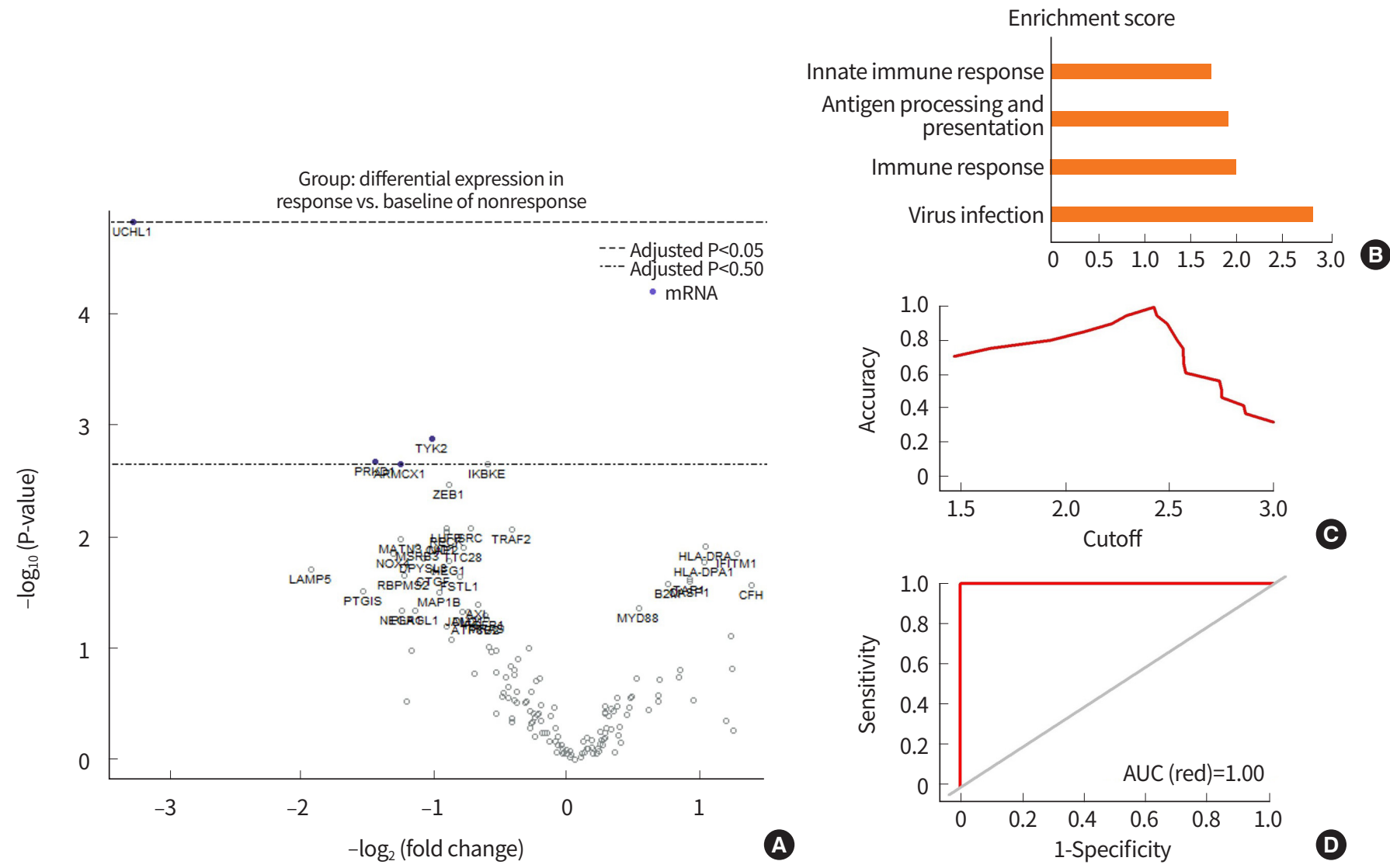

Fig. 1. Signature genes in response to pembrolizumab and IMmunotherapy Against Gastrlc Cancer (IMAGiC) scores. (A) Volcano plot showing results of differentially expressed gene analysis between the response group and the nonresponse group by Response Evaluation Criteria in Solid Tumors (RECIST). (B) Enrichment analysis of differentially expressed genes $\left(P<0.05, \mid \log _{2}\right.$ (fold change) $\geq 1$ ). (C) Cutoff curve of IMAGiC score for dividing the two groups (sensitivity, specificity, and accuracy were all 100\%). (D) Area under the curve (AUC) curve of IMAGiC scores for predicting response to pembrolizumab (AUC=1.00). 


\section{Development of IMAGiC model to predict responses} for pembrolizumab

To construct a model to predict responses to pembrolizum$a b$, we used a linear regression analysis using mRNA expression levels of four genes selected from DEG. As PD-L1 expression is also an important biomarker for pembrolizumab responses [3,6,12,13], PD-L1 CPS was also used for IMAGiC. To evaluate the performance of IMAGiC, we verified this model in the same patient cohort. All IMAGiC groups matched perfectly to the responses for pembrolizumab. The sensitivity, specificity, and accuracy of the IMAGiC model were calculat-
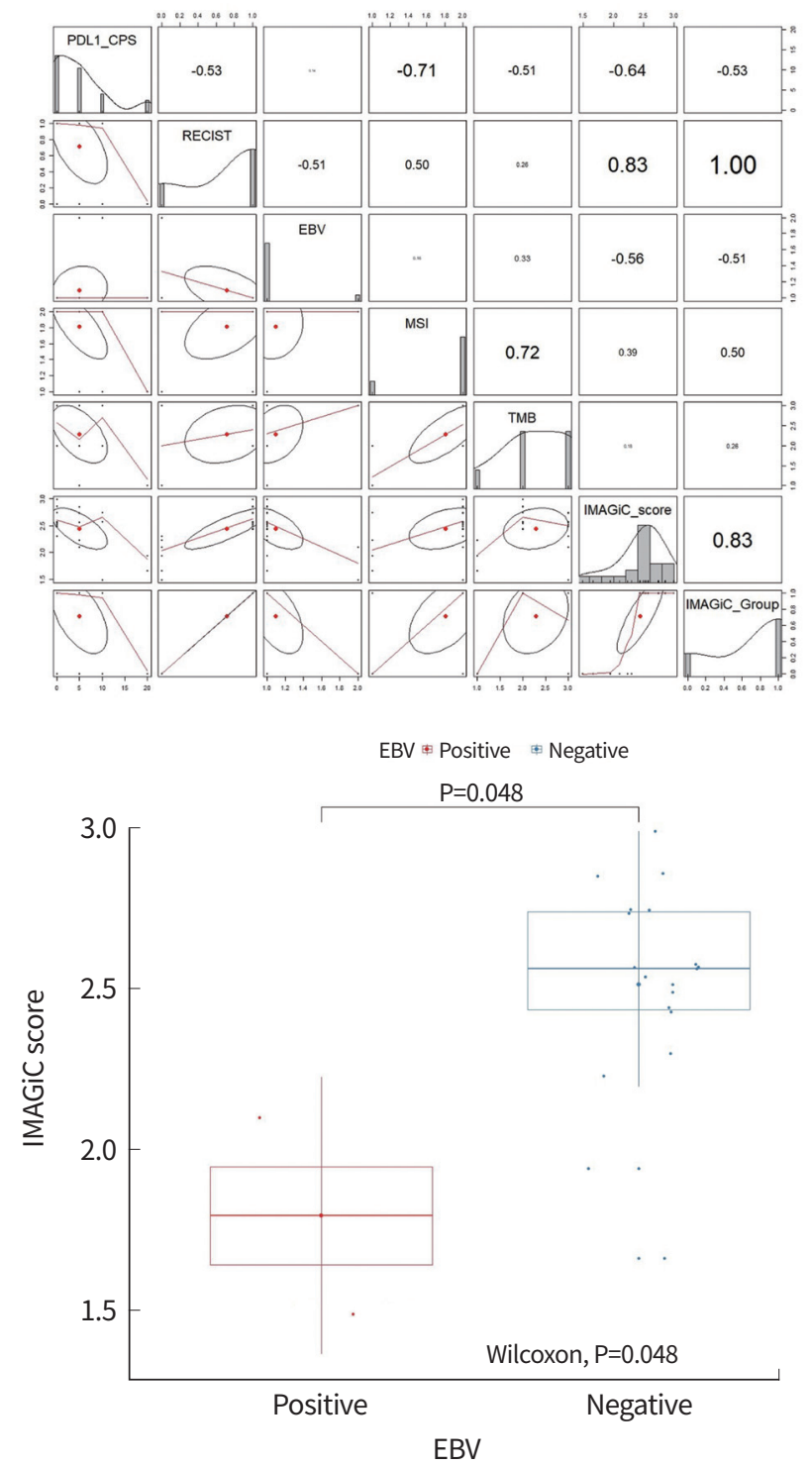

ed using the AUC method, and all of them were 100\% (Fig. $1 C, D)$. To reduce bias of the selected data and improve the performance of the IMAGiC model, we performed 10-fold cross-validation. All 21 samples were used to construct the IMAGiC model and were divided into 10 groups for each cross-validation step; nine of the 10 groups were used for testing and the remaining group was used for validation. The mean RMSE was 1.751.

The IMAGiC group was developed based on the IMAGiC score and RECIST groups for pembrolizumab, and the cutoff value of IMAGiC score was set as 2.5069 using the accuracy
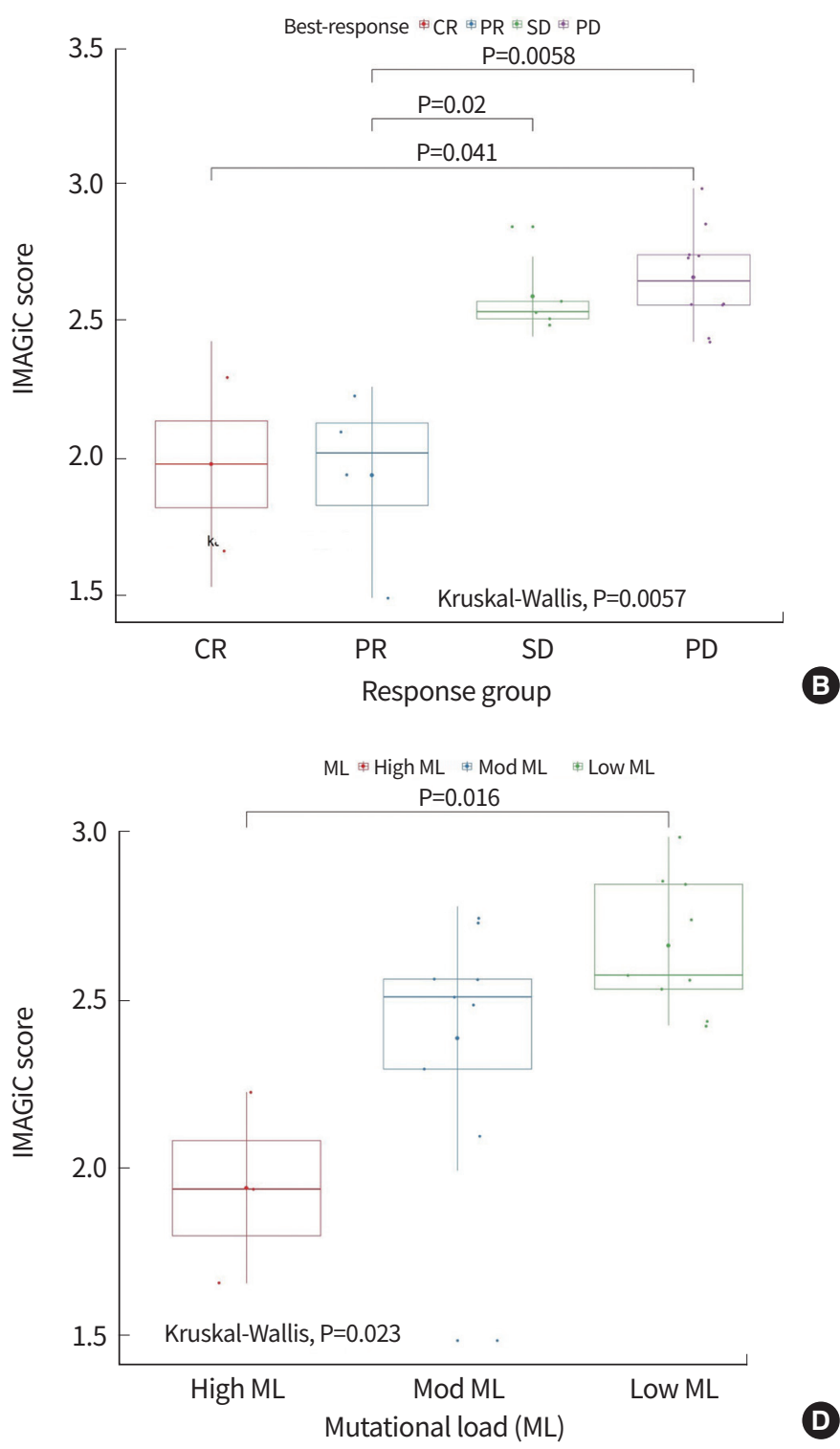

Fig. 2. Associations between IMmunotherapy Against Gastrlc Cancer (IMAGiC) model, Response Evaluation Criteria in Solid Tumors (RECIST) group, Epstein-Barr virus (EBV), and microsatellite unstable (MSI). (A) Correlations between IMAGiC model, RECIST group ( $\left.r^{2}=1.00\right)$, EBV $\left(r^{2}=0.51\right)$, and MSI $\left(r^{2}=0.50\right)$. (B, C, D) Boxplot comparing IMAGiC scores between RECIST groups $(P=0.0057)$, EBV subtypes ( $\left.P=0.048\right)$, and tumor mutation load ( $P=0.023)$. $C R$, complete response; $P R$, partial response; $S D$, stable disease; $P D$, progressive disease; $M L$, mutational load. 
function in the AUC package. Finally, mGC patients were divided into two groups based on IMAGiC: to be responder and to be non-responder. The IMAGiC groups correlated well with RECIST group $\left(r^{2}=0.83\right)$, PD-L1 CPS $\left(r^{2}=0.64\right)$, and EBV infec-

Table 2. Characteristics of responders and non-responders divided by IMAGiC in TCGA

\begin{tabular}{lccc}
\hline IMAGiC group & $\begin{array}{c}\text { Responders } \\
(\mathrm{n}=125)\end{array}$ & $\begin{array}{c}\text { Non-responders } \\
(\mathrm{n}=135)\end{array}$ & P-value \\
\hline Sex & & & 0.7102 \\
Male & $74(46.8)$ & $84(53.2)$ & \\
Female & $51(50.0)$ & $51(50.0)$ & \\
TCGA subtype & & & $1.21 \mathrm{E}-04$ \\
EBV & $16(66.7)$ & $8(33.3)$ & \\
MSI & $37(62.7)$ & $22(37.3)$ & \\
CIN & $60(47.6)$ & $66(52.4)$ & \\
GS & $12(23.5)$ & $39(76.5)$ & \\
MSI & & & \\
MSI-H & $37(62.7)$ & $22(37.3)$ & \\
MSS & $88(43.8)$ & $113(56.2)$ & \\
EBV & & & \\
Positive & $16(66.7)$ & 8.0894 \\
Negative & $109(46.2)$ & $127(53.8)$ & \\
Total mutation rate ${ }^{\mathrm{a})}$ & $21.2401 \pm 34.4094$ & $12.6771 \pm 25.5755$ & $1.19 \mathrm{E}-05$
\end{tabular}

Values are presented as number (\%) or mean \pm standard deviation. IMAGiC, IMmunotherapy Against Gastrlc Cancer; TCGA, The Cancer Genome Atlas; EBV, Epstein-Barr virus; MSI, microsatellite unstable; $\mathrm{CIN}$, chromosomal instability; GS, genomically stable; MSI-H, microsatellite instability-high; MSS, microsatellite stable.

${ }^{a)}$ Statistical analysis was performed after removing not available data. tion status $\left(r^{2}=0.56\right)$. The IMAGiC score was significantly associated with RECIST groups $(P=0.0057)$, EBV status $(P=$ 0.048), and TML ( $P=0.023$ ) (Fig. 2).

Table 3. Characteristics of responders and non-responders divided by IMAGiC in ACRG

\begin{tabular}{lccc}
\hline IMAGiC group & $\begin{array}{c}\text { Responders } \\
(\mathrm{n}=118)\end{array}$ & $\begin{array}{c}\text { Non-responders } \\
(\mathrm{n}=182)\end{array}$ & P-value \\
Sex & & & 0.0396 \\
$\quad$ Male & $87(43.7)$ & $112(56.3)$ & \\
Female & $31(30.7)$ & $70(69.3)$ & \\
ACRG subtype & & & $1.96 \mathrm{E}-08$ \\
MSI & $37(54.4)$ & $31(45.6)$ & \\
EMT & 0 & $46(100)$ & \\
MSS/TP53+ & $31(39.2)$ & $48(60.8)$ & \\
MSS/TP53- & $50(46.7)$ & $57(53.3)$ & \\
MSI & & & \\
MSI-H & $37(54.4)$ & $31(45.6)$ & \\
MSS & $81(34.9)$ & $151(65.1)$ & \\
EBV & & & \\
Positive & $3(16.7)$ & $15(83.3)$ & \\
Negative & $106(41.3)$ & $151(58.8)$ & \\
NA & $9(36.0)$ & $16(64.0)$ & \\
\hline
\end{tabular}

Values are presented as number (\%).

IMAGiC, IMmunotherapy Against Gastrlc Cancer; ACRG, Asian Cancer Research Group; MSI, microsatellite unstable; EMT, epithelial-mesenchymal transformation; MSS, microsatellite stable; TP53, tumor protein p53; MSI-H, microsatellite instability-high; EBV, Epstein-Barr virus; NA, not available.

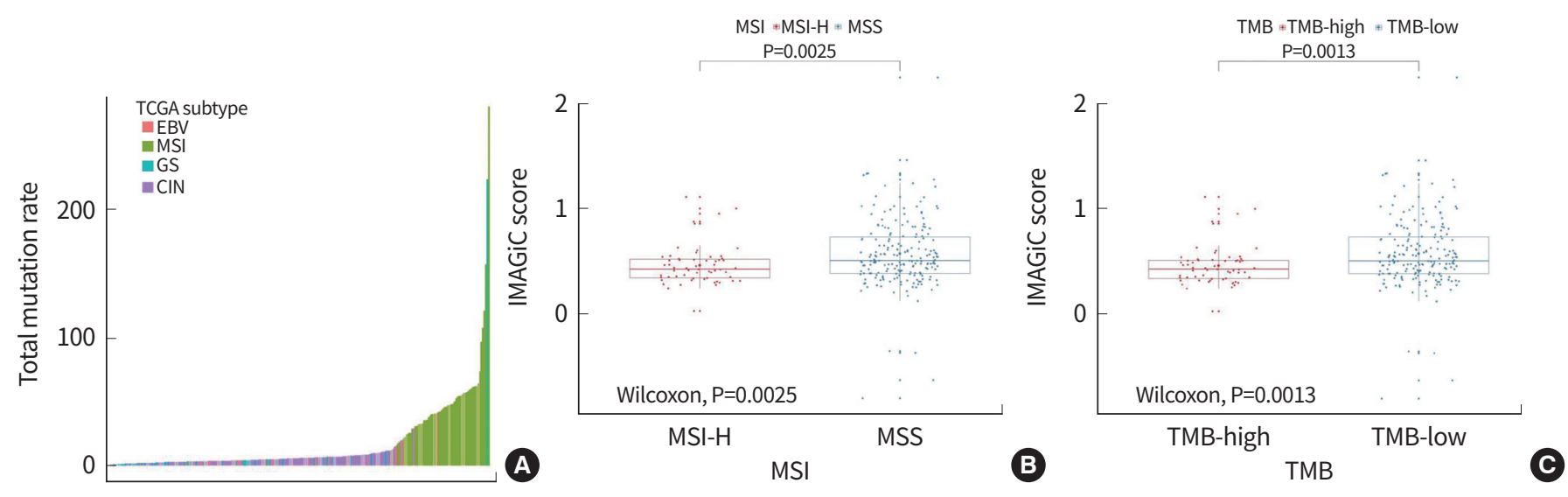

Fig. 3. Analyses of IMmunotherapy Against Gastrlc Cancer (IMAGiC) model in The Cancer Genome Atlas (TCGA) cohorts. (A) Mutational spectrum in TCGA cohorts. The $x$-axis represents tumor identity. (B) Boxplot comparing IMAGiC scores between microsatellite unstable (MSI) subtypes $(P=0.0025)$. (C) Boxplot comparing IMAGiC scores and hypermutated groups defined by tumor mutational load ( $P=0.0013)$. $E B V$, Epstein-Barr virus; GS, genomically stable; CIN, chromosomal instability; MSI-H, microsatellite instability-high; MSS, microsatellite stable; $\mathrm{TMB}$, tumor mutational burden. 


\section{Analyses of IMAGiC in TCGA and ACRG cohorts}

As PD-1 blockades have been shown to be effective for MSI $[6,14]$ and EBV-positive tumors [15-17] as well for tumors with high TMB $[18,19]$, we applied the IMAGiC prediction model for TCGA RNA sequencing data $(n=260)[10]$ and ACRG mRNA expression array results $(n=300)$ [20].

In the TCGA group, there were 59 cases (22.7\%) with MSI, 24 (9.2\%) with EBV-positive, and 61 (23.5\%) with TMB-high subtype GC. The total mutation rates were distinct in four molecular subtypes (Fig. 3A). IMAGiC was significantly associated with TCGA molecular subtype ( $P=1.21 \mathrm{E}-04)$, $\mathrm{MSI}(\mathrm{P}=0.01)$, EBV $(P=0.09)$, and TMB status $(P=1.19 E-05)$ (Table 2). Many patients with MSI- or EBV-positive GC were classified as responders by IMAGiC. The IMAGiC scores in GC associated with MSI and TMB were significantly higher than those in the microsatellite stable (MSS) and no-TMB groups (Fig. 3B, C).

In ACRG, 68 (22.7\%) were MSI and 18 (6.0\%) were EBV-positive subtypes (Table 3 ). We found that IMAGiC groups were associated with recurrence-free survival, ACRG molecular subtype ( $P=1.96 \mathrm{E}-08)$, and MSI ( $P=0.006)$ (Fig. 4).
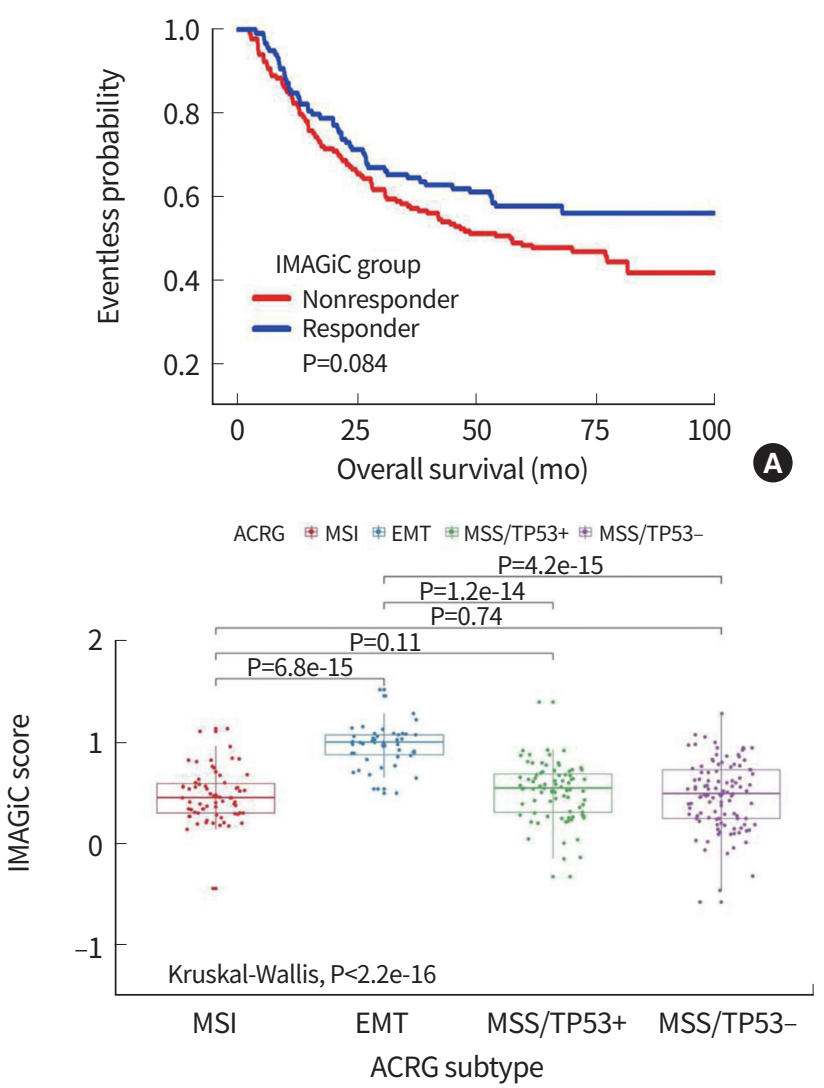

\section{Reproduction and validation of IMAGiC with qRT-PCR} To assess the reproducibility of the IMAGiC model with different technology platforms for use in clinical practice, we developed qRT-PCR using mRNA from 24 patients from the same cohort. The IMAGiC groups by qRT-PCR also highly correlated with the RECIST group $\left(r^{2}=0.82\right)$, EBV $\left(r^{2}=0.48\right)$, and MSI $\left(r^{2}=0.66\right)$ (Supplementary Fig. 3). The precision of reproduction of IMAGiC was $87.5 \%$ (positive predictive value, $87.5 \%$; negative predictive value, $12.5 \%$ ).

To validate IMAGiC qRT-PCR in other patients with $\mathrm{MGC}$, we used 17 samples from the ongoing trial with nivolumab (Opdivo, Bristol-Myers Squibb Company Inc., New York, NY, USA). All cases were EBV-negative, and most of them (94.1\%) were MSS and PD-L1 CPS negative (Table 4). Like recent clinical trials and studies in which MSI, EBV-positivity, and positive PDL1 CPS were associated with response to pembrolizumab [6], most of the cases were classified into non-responder based on RECIST group. To investigate whether the IMAGiC qRT-PCR model was as accurate as the original, we calculated IMAGiC scores using this nivolumab cohort and the precision was
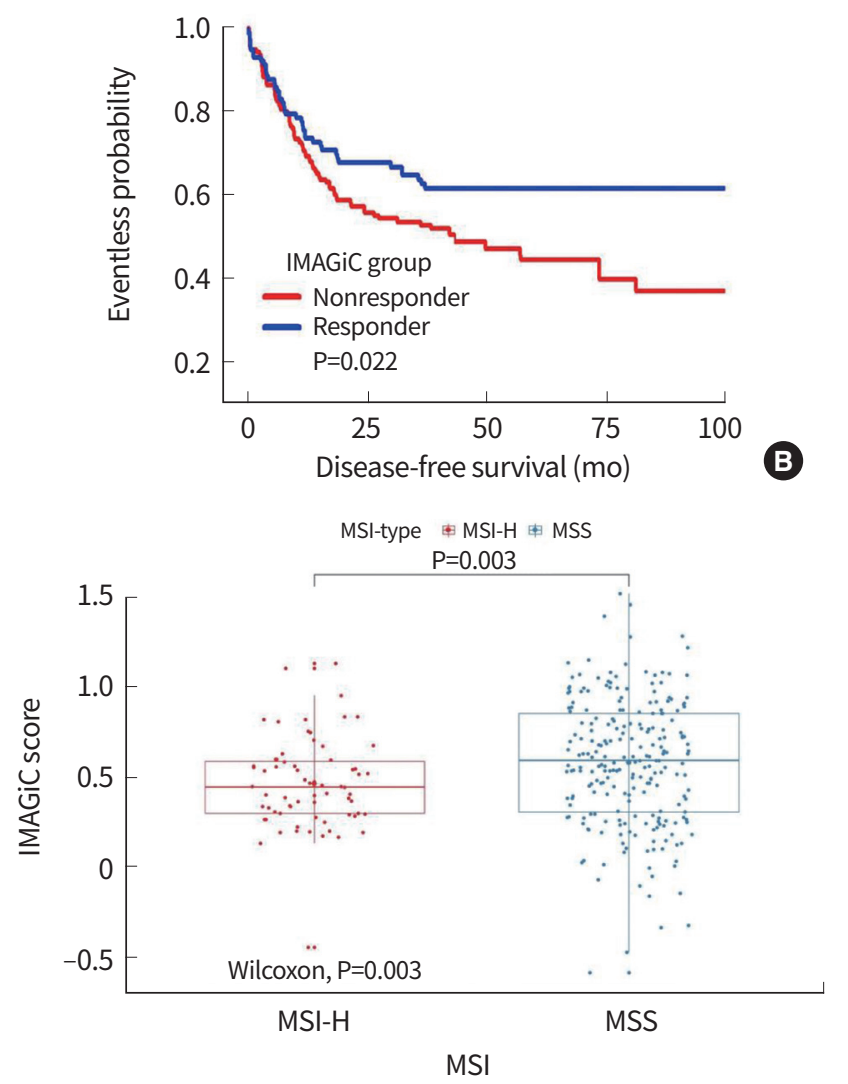

Fig. 4. Analyses of IMmunotherapy Against Gastrlc Cancer (IMAGiC) model in Asian Cancer Research Group (ACRG) cohorts. (A, B) Overall survival (OS) and disease-free survival (DFS) differences between IMAGiC groups in ACRG cohorts ( $P=0.084$ in OS and $P=0.022$ in DFS). (C, D) Boxplot comparing IMAGiC scores between ACRG subtypes $(P<0.001)$ and MSI subtypes $(P=0.003)$. MSI, microsatellite unstable; EMT, epithelial-mesenchymal transformation; MSS, microsatellite stable; TP53, tumor protein p53; MSI-H, microsatellite instability-high 
PRECISION AND FUTURE MIEDICINE

Biomarker of response to immunotherapy

Table 4. Evaluation results of IMAGiC model based on quantitative real-time polymerase chain reaction using validation cohorts

\begin{tabular}{|c|c|c|c|c|c|c|}
\hline Sample & TMB & MSI & EBV & PD-L1 CPS & IMAGiC score & IMAGiC group \\
\hline 1 & 9.27 & MSS & Negative & 0 & 0.9722 & Non-responder \\
\hline 2 & 10.93 & MSS & Negative & 0 & 0.3100 & Non-responder \\
\hline 3 & 3.37 & MSS & Negative & 0 & 1.3076 & Non-responder \\
\hline 4 & 1.68 & MSS & Negative & 0 & 0.3926 & Non-responder \\
\hline 5 & 4.2 & MSS & Negative & 0 & 0.3314 & Non-responder \\
\hline 6 & 3.35 & MSS & Negative & 0 & 0.3534 & Non-responder \\
\hline 7 & 0 & MSS & Negative & 0 & 0.3726 & Non-responder \\
\hline 8 & 3.38 & MSS & Negative & 0 & 0.2417 & Non-responder \\
\hline 9 & 6.75 & MSS & Negative & 0 & 0.2220 & Non-responder \\
\hline 10 & 5.04 & MSS & Negative & 0 & 0.4416 & Non-responder \\
\hline 11 & 2.54 & MSS & Negative & 0 & 1.6643 & Non-responder \\
\hline 12 & 2.52 & MSS & Negative & 0 & 0.2412 & Non-responder \\
\hline 13 & 5.05 & MSS & Negative & 0 & 1.0175 & Non-responder \\
\hline 14 & 5.9 & MSS & Negative & 0 & 0.3310 & Non-responder \\
\hline 15 & 2.53 & MSS & Negative & 0 & 0.7392 & Non-responder \\
\hline 16 & 10.11 & MSI-H & Negative & 1 & 0.3268 & Non-responder \\
\hline 17 & 8.43 & MSS & Negative & 0 & 0.8758 & Non-responder \\
\hline
\end{tabular}

IMAGiC, IMmunotherapy Against Gastrlc Cancer; TMB, tumor mutation burden; MSI, microsatellite unstable; EBV, Epstein-Barr virus; PD-L1, programmed death ligand 1; CPS, combined positive score; MSS, microsatellite stable; MSI-H, microsatellite instability-high.

$100 \%$ (positive predictive value, 100\%; negative predictive value, $0 \%$ ) although the number of cases was small.

\section{DISCUSSION}

Identifying accurate biomarkers predicting response to immunotherapy is crucial for precision oncology. Here, we developed an IMAGiC model that highly correlated with RECIST, MSI, TMB, and molecular subtype of GC. To easily apply this prediction model in the clinic, a qRT-PCR-based IMAGiC model was developed and reproduced these results.

Molecular characterization study showed that approximately $34 \%$ of GCs exhibit a relatively high TMB including, but not limited to, tumors with MSI [10]. In addition to GC with EBV-associated and MSI, tumors with high TMB also responded well to pembrolizumab, a selective humanized monoclonal antibody designed to bind to PD-1 that blocks the interaction between PD-1 and its ligands such as PD-L1 [21]. However, the high numbers of non-responders and the emergence of resistance in patients who do initially respond are significant challenges in the field of cancer immunotherapy [5]. A recent molecular characterization study of patients with $\mathrm{mGC}$ treated with pembrolizumab showed that MSI, EBV, PD-L1
CPS, and TMB were associated with responses [6]. In addition, in a cohort of patients with $\mathrm{MGC}$ treated with nivolumab, a fully human immunoglobulin G4 (IgG4) monoclonal antibody against PD-1, responses were observed in patients with at least one of the following factors: presence of the dMMR biomarker, high TMB, EBV-positive status, and PD-L1-positive status in tumor cells. Given that elevated PD-L1 expression is frequently observed in $\mathrm{GC}$ with an elevated host immune response, tumors with MSI/EBV-associated/high TMB are associated with a favorable prognosis $[22,23]$ and predict responses to pembrolizumab in GC [6]. To prove this, we built a predictor of responses to pembrolizumab after analyzing the mRNA expression and PD-L1 CPS data of 25 patients. We focused on four genes related to immune and mesenchymal transformation using machine learning technology. Based on these findings, the prediction scores were calculated, with lower scores predicting higher chances of responses. Reassuringly, by examining an additional 17 tumor samples from patients treated with nivolumab, we found that IMAGiC again predicted the responses of patients to treatment. Additionally, we found that IMAGiC was significantly associated with recurrence-free survival in ACRG datasets.

Pembrolizumab is one of the well-known targeted ICB 
agents for patients with various cancers with overexpression of PD-L1 [2,4,12,24]. Unfortunately, not all patients show a response to pembrolizumab; it is not always successful for patients whose tumors overexpress PD-L1. Moreover, anti-PD-1/PD-L1 antibodies have anecdotally been reported to cause rapid progression of some types of cancers, although the incidence in GC remain unclear [25]. The high populations of non-responders and emergence of hyperprogressive disease pose significant challenges in the field of cancer immunotherapy [26].

Although PD-L1 is obviously an important predictive biomarker for response to immune checkpoint inhibition in GC, MSI, dMMR, and high TMB have demonstrated potential roles as positive predictive biomarkers [6,27]. In the present study, we combined PD-L1 CPS and signature gene set data and developed an IMAGiC model to predict response to pembrolizum$a b$ in patients with $\mathrm{mGC}$, and those results were validated in patients with $\mathrm{mGC}$ treated with nivolumab. Four genes showed significantly different expression levels between the response group and non-response group $(P<0.01)$ and are involved in cell proliferation, innate immune response, protein phosphorylation, and tumor suppression. As the genes comprising IMAGiC were significantly related to immune responses, we could find that the IMAGiC groups correlated well with PD-L1 CPS and EBV infection. In our cohort, all but two patients with MSI GC and all EBV-positive GC were in the responder group, and IMAGiC predicted them to be non-responders.

Given the low response rate, appropriate patients should be selected prior to PD-1/PD-L1 inhibitor therapy. Identifying predictive biomarkers to identify patients most likely to respond to ICB would decrease treatment costs and the likelihood of immune-related adverse events [28]. For our group of pembrolizumab-treated patients, we developed a novel prediction model via machine learning methods based on RECIST response groups, IMAGiC, which is significantly associated with EBV and TMB. PD-L1 expression is generally believed to be a surrogate of pre-existing immune-specific activity and can be upregulated by interferon- $\gamma$ in the tumor microenvironment [29]. However, responses to immune checkpoint inhibition in GC are also related to MSI, EBV, and TMB. With the increased understanding of tumor immune escape, establishing a wide-ranging framework of multiple biomarkers is necessary for patient selection and precision medicine [28].

Although this study confirmed value in predicting response to immune checkpoint inhibitors, the population was small in the discovery cohort $(n=21)$ and validation cohort $(n=17)$. So, further prospective validation is recommended in other cohorts. Moreover, as GC is among the most immunogenic tumors after melanoma, bladder, and lung cancer [30], we are planning to apply IMAGiC model to those immunogenic cancers in ongoing immunotherapy trials.

In conclusion, we developed a combined biomarker, IMAGiC, for predicting responses to pembrolizumab using mRNA levels and PD-L1 CPS. IMAGiC correlated well with EBV, TMB, and MSI and was further validated in other cohorts.

\section{CONFLICTS OF INTEREST}

No potential conflict of interest relevant to this article was reported.

\section{ACKNOWLEDGMENTS}

This work was supported by a grant from the Korean Health Technology R\&D Project, Ministry of Health \& Welfare, Republic of Korea (HI16C1990) and Basic Science Research Program through the National Research Foundation of Korea (NRF) funded by the Ministry of Science and ICT (NRF-2017R1 E1A1A01075005) (NRF-2017R1A2B4012436). Support was also provided by a grant from the 20 by 20 Project of Samsung Medical Center (GFO2190111).

\section{ORCID}

You Jeong Heo https://orcid.org/0000-0002-7966-711X So Young Kang https://orcid.org/0000-0003-2142-2803 Seung Tae Kim https://orcid.org/0000-0001-8647-7157 Won Ki Kang https://orcid.org/0000-0002-7194-2404 Jeeyun Lee https://orcid.org/0000-0002-4911-6165 Kyoung-Mee Kim https://orcid.org/0000-0002-1162-9205

\section{REFERENCES}

1. Torre LA, Bray F, Siegel RL, Ferlay J, Lortet-Tieulent J, Jemal A. Global cancer statistics, 2012. CA Cancer J Clin 2015;65:87-108.

2. Boyiadzis MM, Kirkwood JM, Marshall JL, Pritchard CC, Azad NS, Gulley JL. Significance and implications of FDA approval of pembrolizumab for biomarker-defined disease. J Immunother Cancer 2018;6:35.

3. Ott PA, Bang YJ, Piha-Paul SA, Razak ARA, Bennouna J, Soria JC, et al. T-cell-inflamed gene-expression profile, programmed death ligand 1 expression, and tumor mu- 
tational burden predict efficacy in patients treated with pembrolizumab across 20 cancers: KEYNOTE-028. J Clin Oncol 2019;37:318-27.

4. Fuchs CS, Doi T, Jang RW, Muro K, Satoh T, Machado M, et al. Safety and efficacy of pembrolizumab monotherapy in patients with previously treated advanced gastric and gastroesophageal junction cancer: phase 2 clinical KEYNOTE-059 trial. JAMA Oncol 2018;4:e180013.

5. Terry S, Savagner P, Ortiz-Cuaran S, Mahjoubi L, Saintigny $P$, Thiery JP, et al. New insights into the role of EMT in tumor immune escape. Mol Oncol 2017;11:824-46.

6. Kim ST, Cristescu R, Bass AJ, Kim KM, Odegaard JI, Kim K, et al. Comprehensive molecular characterization of clinical responses to PD-1 inhibition in metastatic gastric cancer. Nat Med 2018;24:1449-58.

7. Auslander N, Zhang G, Lee JS, Frederick DT, Miao B, Moll T, et al. Robust prediction of response to immune checkpoint blockade therapy in metastatic melanoma. Nat Med 2018;24:1545-9.

8. Lee J, Cristescu R, Kim KM, Kim K, Kim ST, Park SH, et al. Development of mesenchymal subtype gene signature for clinical application in gastric cancer. Oncotarget 2017; 8:66305-15.

9. Park C, Cho J, Lee J, Kang SY, An JY, Choi MG, et al. Host immune response index in gastric cancer identified by comprehensive analyses of tumor immunity. Oncoimmunology 2017;6:e1356150.

10. Cancer Genome Atlas Research Network. Comprehensive molecular characterization of gastric adenocarcinoma. Nature 2014;513:202-9.

11. Rizzardi AE, Johnson AT, Vogel RI, Pambuccian SE, Henriksen J, Skubitz AP, et al. Quantitative comparison of immunohistochemical staining measured by digital image analysis versus pathologist visual scoring. Diagn Pathol 2012;7:42.

12. Taube JM, Galon J, Sholl LM, Rodig SJ, Cottrell TR, Giraldo NA, et al. Implications of the tumor immune microenvironment for staging and therapeutics. Mod Pathol 2018; 31:214-34.

13. Rui X, Gu TT, Pan HF, Zhang HZ. Evaluation of PD-L1 biomarker for immune checkpoint inhibitor (PD-1/PD-L1 inhibitors) treatments for urothelial carcinoma patients: a meta-analysis. Int Immunopharmacol 2019;67:378-85.

14. Diaz LA Jr, Le DT. PD-1 blockade in tumors with mismatch-repair deficiency. N Engl J Med 2015;373:1979.

15. Derks S, Liao X, Chiaravalli AM, Xu X, Camargo MC, Solcia E, et al. Abundant PD-L1 expression in Epstein-Barr virus-in- fected gastric cancers. Oncotarget 2016;7:32925-32.

16. lizasa H, Nanbo A, Nishikawa J, Jinushi M, Yoshiyama H. Epstein-Barr virus (EBV)-associated gastric carcinoma. Viruses 2012;4:3420-39.

17. Ma C, Patel K, Singhi AD, Ren B, Zhu B, Shaikh F, et al. Programmed death-ligand 1 expression is common in gastric cancer associated with Epstein-Barr virus or microsatellite instability. Am J Surg Pathol 2016;40:1496-506.

18. Ciombor KK, Goldberg RM. Hypermutated tumors and immune checkpoint inhibition. Drugs 2018;78:155-62.

19. Nebot-Bral L, Brandao D, Verlingue L, Rouleau E, Caron O, Despras E, et al. Hypermutated tumours in the era of immunotherapy: the paradigm of personalised medicine. Eur J Cancer 2017;84:290-303.

20. Cristescu R, Lee J, Nebozhyn M, Kim KM, Ting JC, Wong SS, et al. Molecular analysis of gastric cancer identifies subtypes associated with distinct clinical outcomes. Nat Med 2015;21:449-56.

21. Keytruda (pembrolizumab) injection, for intravenous use [Internet]. Whitehouse Station (NJ): Merck \& Co.; 2015 [cited 2019 Oct 31]. Available from: https://www.accessdata. fda.gov/drugsatfda_docs/label/2015/125514s004s006lbl. pdf.

22. Cho J, Chang YH, Heo YJ, Kim S, Kim NK, Park JO, et al. Four distinct immune microenvironment subtypes in gastric adenocarcinoma with special reference to microsatellite instability. ESMO Open 2018;3:e000326.

23. Cho J, Ahn S, Son DS, Kim NK, Lee KW, Kim S, et al. Bridging genomics and phenomics of gastric carcinoma. Int J Cancer 2019;145:2407-17.

24. Kulangara K, Zhang N, Corigliano E, Guerrero L, Waldroup $\mathrm{S}$, Jaiswal $\mathrm{D}$, et al. Clinical utility of the combined positive score for programmed death ligand-1 expression and the approval of pembrolizumab for treatment of gastric cancer. Arch Pathol Lab Med 2019;143:330-7.

25. Ishii T, Kawazoe A, Shitara K. Dawn of precision medicine on gastric cancer. Int J Clin Oncol 2019;24:779-88.

26. Kurman JS, Murgu SD. Hyperprogressive disease in patients with non-small cell lung cancer on immunotherapy. J Thorac Dis 2018;10:1124-8.

27. Mishima S, Kawazoe A, Nakamura Y, Sasaki A, Kotani D, Kuboki Y, et al. Clinicopathological and molecular features of responders to nivolumab for patients with advanced gastric cancer. J Immunother Cancer 2019;7:24.

28. Yi M, Jiao D, Xu H, Liu Q, Zhao W, Han X, et al. Biomarkers for predicting efficacy of PD-1/PD-L1 inhibitors. Mol Cancer 2018;17:129. 
PRECISION AND FUTURE MIEDICINE

You Jeong $\mathrm{HeO}$, et al.

29. Sun C, Mezzadra R, Schumacher TN. Regulation and function of the PD-L1 checkpoint. Immunity 2018;48:43452.
30. Alexandrov LB, Nik-Zainal S, Wedge DC, Aparicio SA, Behjati S, Biankin AV, et al. Signatures of mutational processes in human cancer. Nature 2013;500:415-21. 


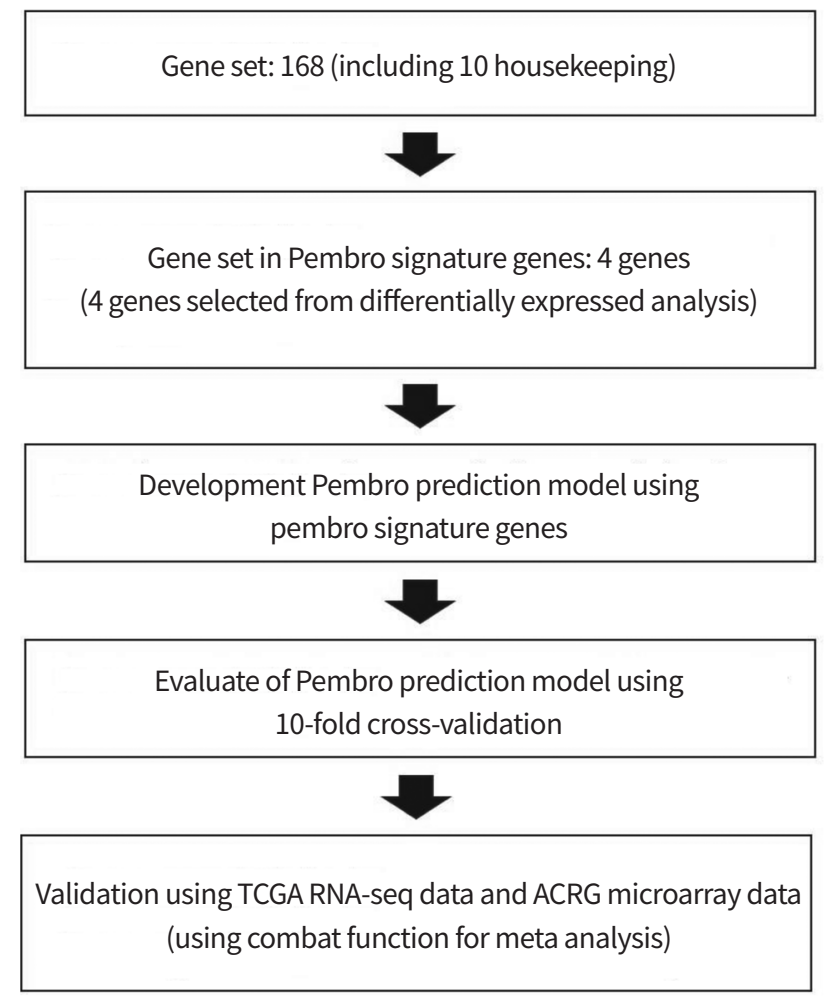

Supplementary Fig. 1. Study design to identify signature genes for pVredicting response to pembrolizumab in gastric cancer. TCGA, The Cancer Genome Atlas; ACRG, Asian Cancer Research Group. 


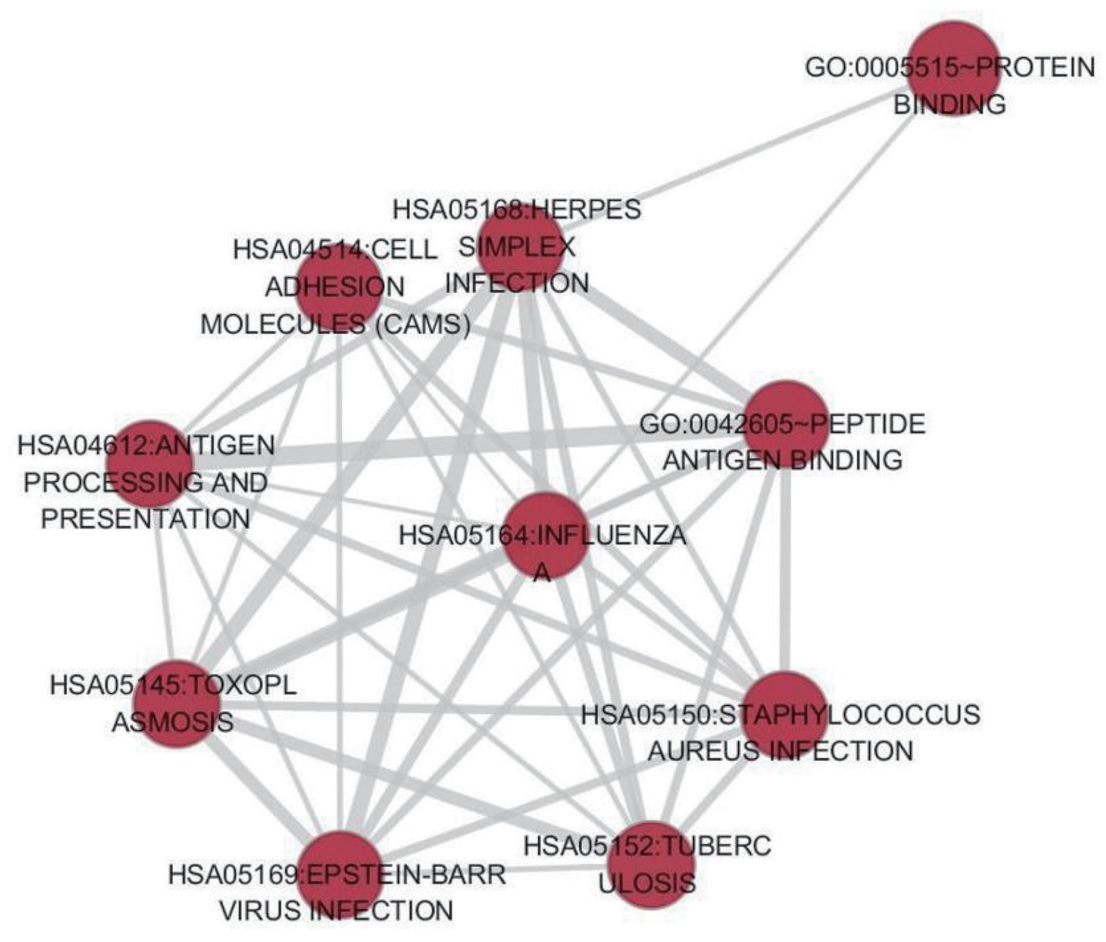

Supplementary Fig. 2. Enrichment map. Enrichment network analysis using differentially expressed genes $\left(P<0.05, \mid \log _{2}\right.$ (fold change) $\left.\mid \geq 1\right)$. 


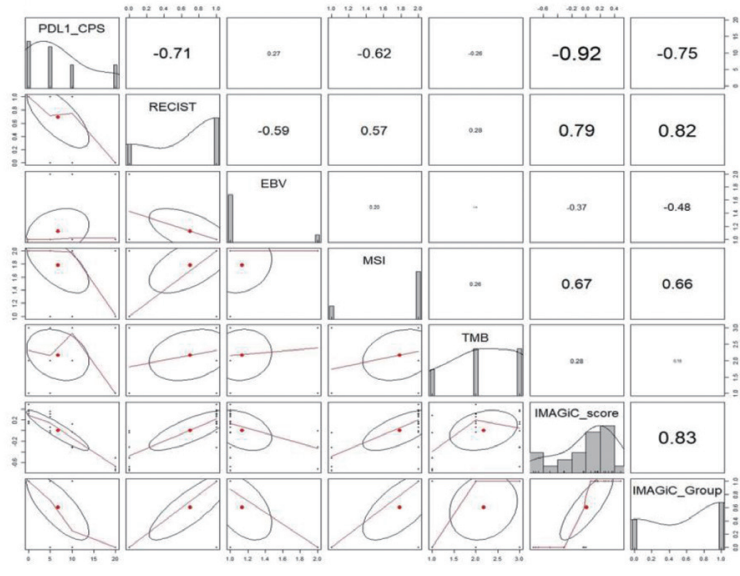

MSI 歹 MSI \$ \$MSS

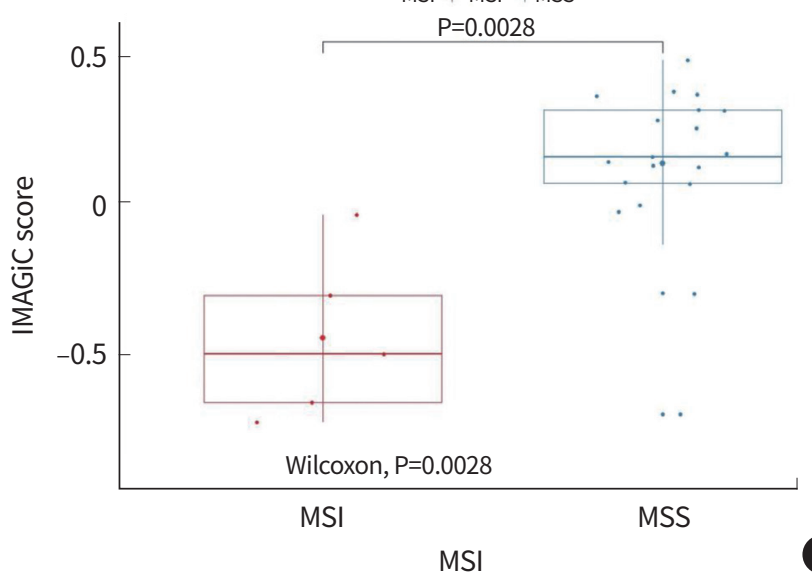

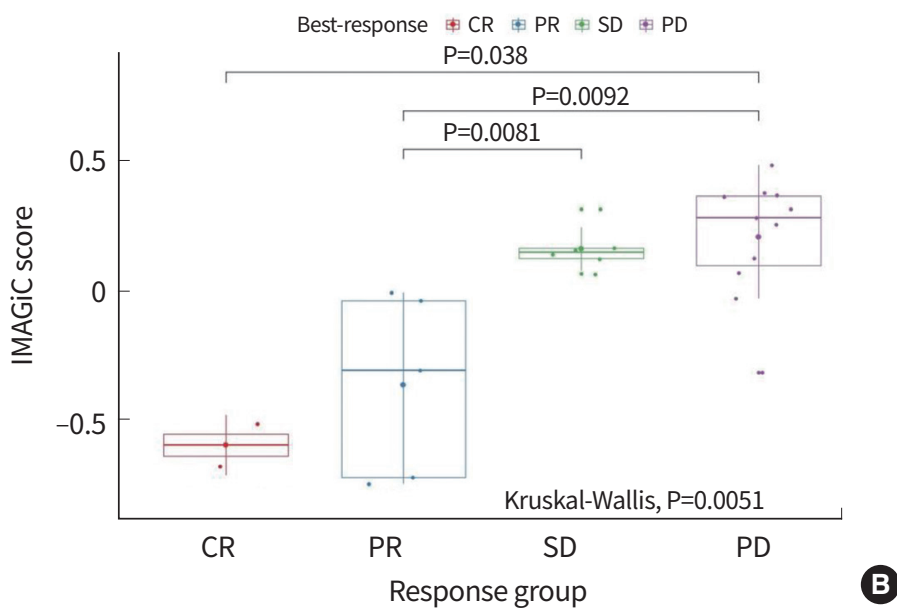

EBV Positive Negative

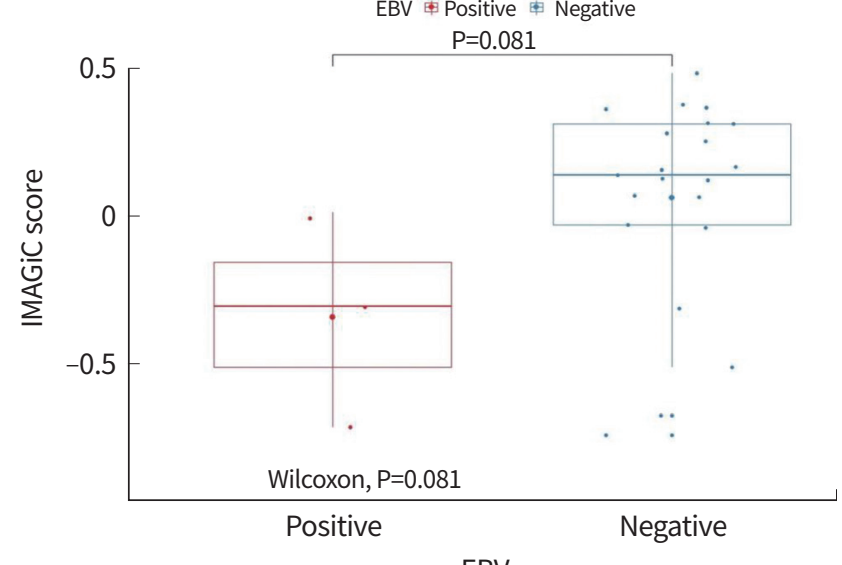

EBV

Supplementary Fig. 3. Reproducibility of IMmunotherapy Against Gastrlc Cancer (IMAGiC) model using a quantitative real-time polymerase chain reaction platform. (A) Correlations between IMAGiC model, Response Evaluation Criteria in Solid Tumors (RECIST) group ( $r^{2}=0.83$ ), Epstein-Barr virus (EBV; $\left.r^{2}=0.49\right)$, and microsatellite unstable (MSI; $\left.r^{2}=0.66\right)$. (B, C, D) Boxplot comparing IMAGiC scores between RECIST groups $(P=0.0051)$, $M S I$ subtypes $(P=0.0028)$, and EBV subtypes $(P=0.081)$. PD-L1, programmed death ligand 1 ; CPS, combined positive score; TMB, tumor mutational burden; $C R$, complete response; $\mathrm{PR}$, partial response; $\mathrm{SD}$, stable disease; PD, progressive disease. 